that mice given microbes from healthy children gained more weight and muscle than did mice with the malnourished microbiomes. The team identified microbial species associated with these gains, and delivering two species to the guts of mice that had malnourished microbiomes boosted the animals' growth. Martin Schwarzer and François Leulier at the University of Lyons, France, and their team found that giving malnourished mice a strain of Lactobacillus plantarum bacteria helped the mice to gain weight by restoring their growth hormone production. In a third study, Gordon and his colleagues identified a sugar in breast milk that promoted growth in mice and piglets harbouring microbes from a malnourished child.

Science http://doi.org/benr (2016); Science 351, 854-857 (2016); Cell http://doi.org/ bcqd (2016)

\section{ANIMAL COGNITION}

\section{Horses read human emotions}

Horses can differentiate between happy and angry human faces.

Researchers previously showed that dogs can identify emotions from human faces. To find out whether horses share this ability, Amy Smith, Karen McComb and their colleagues at the University of Sussex in Brighton, UK, tested the response of domestic horses

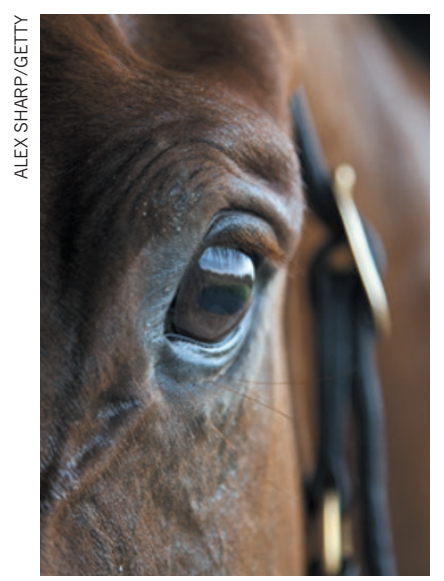

(Equus caballus; pictured) to photographs of human faces with happy or angry expressions. Horses tended to view angry faces with their left eyes - a sign that they were processing the image using the brain's right hemisphere, which is thought to handle negative stimuli. The animals' heart rates also increased more rapidly in response to angry faces than to happy ones.

The ability of horses to recognize human expressions could have evolved during domestication, the authors say. They add that the animals probably also refine this skill during their lifetimes.

Biol. Lett. 12, 20150907 (2016)

\section{PLANETARY SCIENCE}

\section{First super-Earth atmosphere}

The first gases to be identified around an exoplanet that is slightly larger than Earth show that its atmosphere is probably rich in hydrogen and carbon.

Angelos Tsiaras of University College London and his colleagues used a camera on the Hubble Space Telescope to probe the planet 55 Cancri e, which has a radius twice that of Earth, lies 12 parsecs (about 40 light years) away and orbits close to its host star.

The team found that the planet's atmosphere contains no water vapour but does have hydrogen, probably in the form of hydrogen cyanide, which is an indicator of carbon-rich atmospheres. The high level of carbon suggests exotic chemistry around the planet. Astrophys. J. in the press; preprint at http://arxiv.org/ abs/1511.08901v2 (2016)

\section{CLIMATE CHANGE}

\section{Climate risks of low-carbon power}

A transition to power plants that capture and store carbon could increase water use, probably leading to shortages in a major UK river basin as early as the 2030 s.

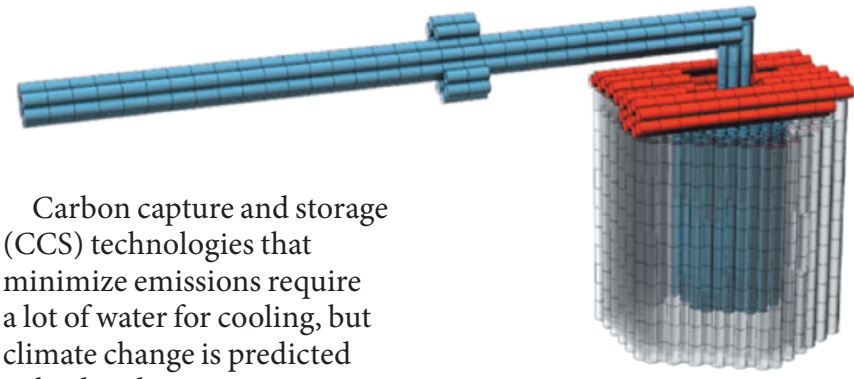

to lead to drier summers in the United Kingdom. Edward Byers of Newcastle University, UK, and his colleagues used climate and hydrology models to analyse scenarios in which electricity production rises by $55 \%$ by 2040 as CCS technologies are fully adopted in the River Trent basin - the largest UK inland source of cooling water for power generation. The team projected declining river flows alongside rising demand for water from the power sector.

The researchers found that CCS power plants might become less reliable in the future when the river runs low, unless the most waterefficient technologies are used.

Environ. Res. Lett. 11, 024011 (2016)

Nanorotor made
of DNA

Researchers have made a nanometre-scale rotor out of 3D fragments of DNA.

Hendrik Dietz and his colleagues at the Technical University of Munich, Germany, designed the fragments to self-assemble into a rotor that looks like a helicopter blade (pictured). It spins around on an axle and is clamped in place by two other DNA units. To stop the blade from rotating freely, the team used docking sites on the inside of the clamp units so that the rotor was held in place. When the ion concentration of the solution containing the rotor changed, the rotor was released and could spin owing to Brownian motion.

The researchers say this action mimics that of an enzyme in the body that acts like a rotary machine, and claim that their nanomachine is more structurally complex than previous ones.

Sci. Adv. 2, e1501209 (2016)

\section{ROBOTICS}

\section{Microbots dance in the light}

Tiny soft robots can swim, spin and make other complex motions in response to light.

Microbots - micrometrescale robots - could one day be used to deliver medicines or to collect data inside the body. But most microbots are made of rigid materials, which limit them to simple back-and-forth motions, usually in response to chemical or magnetic forces. To develop microbots that are capable of more-versatile movement, Peer Fischer at the Max Planck Institute for Intelligent Systems in Stuttgart, Germany, and his colleagues designed soft, flexible ones using a liquidcrystal elastomer that was both rubbery and responsive to light.

By sweeping bands of light across disc- and rodshaped microbots, the team induced wave-like swimming motions similar to those used by single-celled organisms. Different patterns of light also made the disc-shaped microbots spin, reverse their spinning direction or move around a path in the shape of a square.

Nature Mater. http://doi.org/bcjk (2016)

\section{DNATURE.COM}

For the latest research published by Naturevisit:

www.nature.com/latestresearch 